Review

\title{
Long Period Gratings in Photonic Crystal Fibers
}

\author{
Jian JU and Wei JIN
}

Department of Electrical Engineering, The Hong Kong Polytechnic University, Hung Hom, Kowloon, Hong Kong, China

*Corresponding author: Wei JIN_Ｅ-mail: eewjin@polyu.edu.hk

\begin{abstract}
The authors review the recent advances in fabricating long-period gratings (LPGs) in photonic crystal fibers (PCFs). The novel light-guiding properties of the PCFs allow the demonstration of novel sensors and devices based on such LPGs. The sensitivity of these PCF LPGs to temperature, strain and refractive index is discussed and compared with LPGs made on conventional single-mode fibers. In-fiber devices such as tunable band rejection filters, Mach-Zehnder interferometers are discussed.
\end{abstract}

Keywords: Photonic crystal fiber, long-period grating, optical fiber sensor

\section{Introduction}

A long-period grating (LPG) couples light resonantly from the fundamental core mode to a co-propagating higher order core or cladding mode. It is usually formed by periodically perturbing the refractive index or geometry longitudinally along the length of an optical fiber, with a typical period from several hundreds of micrometers to $1 \mathrm{~mm}$. The co-propagating cladding modes are eventually lost from the fiber, which leads to attenuation bands in the transmission spectrum. The resonant wavelength $\lambda_{\text {res }}$ of an LPG with period $\Lambda$ is determined by the phase matching condition [1]:

$$
\lambda_{\mathrm{res}}=\left(n_{\mathrm{co}}-n_{\mathrm{cl}, m}\right) \cdot \Lambda
$$

where $n_{\mathrm{co}}$ and $n_{\mathrm{cl}, m}$ are the effective indices of the fundamental core mode and the $m$ th higher-order or cladding mode, respectively.

Photonic crystal fiber (PCF) is a new type of optical fiber with periodic array of air-holes running along its length [2-3]. PCFs have attracted great interest during the past decade because of its unique properties such as endless single-mode, large mode area, high nonlinearity, and air-core guiding. Such properties are due to the complex index profile and special properties of the photonic crystal cladding. The emergence of PCFs has resulted in LPGs inscribed in them with novel properties. For example, the resonant wavelength of an LPG written in a PCF decreases with the gratings period $\Lambda$, which is contrary to that in a conventional single mode fiber (SMF).

In this paper, we overview various techniques for fabricating LPGs in PCFs and discuss the applications of these LPGs in sensing and photonic devices.

\section{LPG fabrications in PCF}

The first demonstration of LPG in PCF can be traced back to 2002, in which gratings are formed by periodically deforming the air-holes in the cladding when it is subjected to $\mathrm{CO}_{2}$ laser irradiation [4]. As PCFs are typically made of a single material (silica) that is not photosensitive, inscription of PCFs by direct ultra violet (UV) irradiation is difficult. Therefore, non-photochemical inscription techniques

Received: 12 October 2010 / Revised version: 30 December 2010

(C) The Author(s) 2011.This article is published with open access at Springerlink.com 
are commonly used. These may include $\mathrm{CO}_{2}$ laser irradiation [4-10], electrical arc discharge [11-13], applying mechanical pressure [14-16], and acousto-optic interaction [17-20]. It was until 2007 that photochemical recording of LPG in PCF by using a high-intensity femtosecond UV radiation was reported [21].

$\mathrm{CO}_{2}$ laser has been proved to be a versatile and effective tool for micro-machining and post-processing of optical fibers. It has been widely used for LPG inscription in optical fibers since its first demonstration in 1998 [22]. With a point-by-point writing technique, very compact $(2.8 \mathrm{~mm})$ and deep-notched $(-31.5 \mathrm{~dB})$ LPGs have been fabricated in PCF [5]. There're two main factors that facilitate the formation of LPG in PCF: refractive index change due to the residual stress relaxation and waveguide geometry variation. For the index-guiding PCF where the central core is solid, LPG could be formed solely by the stress relaxation without deforming the air-holes [6]. The residual stress, which is formed during the drawing process of the fiber, will influence the refractive indices of the core and cladding via the photo-elastic effects. The periodic release of the residual stress by a focused $\mathrm{CO}_{2}$ laser or an electric arc discharge will introduce the refractive index modulation along the fiber and form LPG in PCFs. However, the stress release is believed to play a less significant role in the formation of LPG in a hollow-core photonic band gap (PBG) PCF [9], as over 95\% of the mode power is in air, and the LPG is formed mainly by the period deformation air-holes at the laser treatment spots.

LPG formation mechanisms in PCF by arc discharge are very similar to that by $\mathrm{CO}_{2}$ lasers [11-13]. However, the reported number of the grating periods that is needed to achieve a comparable attenuation band is much larger than that of the $\mathrm{CO}_{2}$ laser irradiation technique. This may be due to the relatively smaller modification of the refractive index and geometrical deformation introduced by the arc-discharge method.

Mechanical pressure has provided another direct and flexible means for LPG inscription in PCF [14-16]. The strength and wavelength of the resonant peak can be tuned simply by adjusting the grating period and the pressure applied on the PCF. However, the coupling was found to be highly polarization-dependent and depended on the angular position where stress was applied [15]. The highly polarization-dependent broadband coupling was observed due to the unique beat-length dispersion between the core-mode and cladding-mode, which could find potential applications in wide-band polarization dependent loss (PDL) compensation.

Acousto-optical interaction can also cause mode coupling between different modes if phase matching conditions are satisfied. The first demonstration of exciting cladding modes acoustically in PCF was reported by Diez et al. [17], and later Haakestad et al. used a similar means to investigate the propagation constants of different modes supported by a PCF [18]. The LPG strengths of these preliminary works are relatively weak compared with those LPGs in conventional single mode fibers. The acoustically induced LPGs were also demonstrated in a solid core PBG PCF that was formed by filling higher refractive index fluid into the air holes of an otherwise index-guiding PCF [19]. The mode propagation constants of this type of PCF are highly dispersive, resulting in narrower notch-filter bandwidth. We recently demonstrated acoustically induced notch filter by using broadband two-mode PCF [23] and achieved an ultra-wide wavelength tuning range of over $1000 \mathrm{~nm}$ [20]. As indicated by these examples, PCFs provide new promising platforms for developing novel tunable acousto-optic devices by combining the tunability of the acousto-optic interaction with the unique mode properties of the PCFs.

The PCF-based LPGs fabricated by using $\mathrm{CO}_{2}$ or femtosecond laser have demonstrated high strength $(>20 \mathrm{~dB})$ and low insertion losses. They are also 
compact in size $(1 \mathrm{~cm}-3 \mathrm{~cm})$ and can be readily integrated into conventional optical fiber systems. This makes them promising candidates for applications in sensing and telecommunication.

\section{PCF LPG sensors}

LPGs in PCFs have a variety of novel properties. The most prominent one is that they have small temperature sensitivity, as compared with their SMF counterparts. Table 1 lists the strain sensitivity and temperature sensitivity of PCF-based LPGs fabricated by different techniques: $\mathrm{CO}_{2}$ laser, electric arc discharge, and femtosecond laser. The sensitivity of SMF-based LPGs is also listed in the table for comparison.

Table 1 Strain sensitivity and temperature sensitivity of LPG in PCF by different methods.

\begin{tabular}{|c|c|c|c|c|c|c|}
\hline Inscription method & Period $(\mu \mathrm{m})$ & Length (mm) & Wavelength (nm) & $d \lambda / d \varepsilon(\mathrm{pm} / \mu \varepsilon)$ & $d \lambda / d T\left(\mathrm{pm} /{ }^{\circ} \mathrm{C}\right)$ & Ref. \\
\hline $\mathrm{CO}_{2}$ laser & 530 & 3.18 & 1299.59 & -0.192 & 10.9 & {$[6]$} \\
\hline $\mathrm{CO}_{2}$ laser & 410 & 16.4 & 1546.87 & -7.6 & 3.91 & {$[8]$} \\
\hline $\mathrm{CO}_{2}$ laser & 410 & 16.4 & 1548.38 & -0.31 & 3.84 & {$[8]$} \\
\hline Arc discharge & 400 & 37.6 & 1668 & -2.5 & 3.4 & [12] \\
\hline Arc discharge & 1000 & 19.0 & 1403 & -2.08 & 2.2 & [12] \\
\hline Femtosecond laser & 500 & 10 & 1630 & & 249 & [21] \\
\hline Femtosecond laser & 600 & 12 & 1681 & & 293 & [21] \\
\hline UV irradiation* & 320 & & 1573.1 & 7.31 & 46 & [25] \\
\hline $\mathrm{CO}_{2}$ laser* & & & 1526.5 & -0.45 & 58 & [38] \\
\hline Arc discharge* & 540 & & 1580 & $\sim 1.5$ & $66-73$ & [39] \\
\hline Femtosecond laser* & 500 & 10 & 1650 & & 38 & [21] \\
\hline
\end{tabular}

* Conventional single-mode fiber based LPG.

Apparently PCF-based LPGs have very small temperature sensitivity, which makes them useful for strain measurement with negligible temperature cross-sensitivity. Zhao et al. demonstrated a temperature insensitive strain sensor which employed only a distributed feedback (DFB) laser and an optical power meter [24]. The strain sensitivity can be further enhanced, without sacrificing the temperature insensitivity, by introducing periodic grooves carved along the surface of a large mode area (LMA) PCF [8]. The strain sensitivity obtained is $-7.6 \mathrm{pm} / \mu \varepsilon, 25$ times higher than the one without visible grooves.

The very small values of LPG temperature sensitivity in PCF by using $\mathrm{CO}_{2}$ laser irradiation or arc discharge make it unsuitable for temperature sensing. However, it is possible to increase its temperature sensitivity to about $-300 \mathrm{pm} /{ }^{\circ} \mathrm{C}$ by using two-photon absorption (TPA) of femtosecond UV radiation [21]. And its sensitivity is considerably higher than that of SMFs.

LPGs in index-guiding PCF exhibit higher sensitivity to surrounding refractive index (SRI) than those fabricated in standard SMFs (typically about $-60 \mathrm{~nm} / \mathrm{RIU}$ ) and show a red wavelength shift instead of the blue shift observed in SMF [25]. The sensitivity of PCF-based LPG is nonlinear and reaches its maximum when SRI approaches that of the cladding with a shift around $440 \mathrm{~nm} / \mathrm{RIU}$ [26]. There's no appreciable wavelength shift for refractive indices greater than that of silica because the total internal reflection can not occur at cladding-air interface. But the depth of the attenuation band grows with the increase of SRI, which can be attributed to the Fresnel reflections at the cladding-SRI interface. Recently Rindorf et al. reported a maximum sensitivity of $1500 \mathrm{~nm} / \mathrm{RUI}$ around a refractive index of 1.33 where methanol is directly infiltrated into air-holes of an index-guiding PCF [27]. Unlike previous experiments, where PCF 
is immersed into the refractive index liquid, the infiltration into the air-holes allows direct and strong interaction between the light field and liquid. Hence, very high sensitivity was obtained.

The LPG in index-guiding PCF was also demonstrated for bend sensing and the responses to micro-bending are different for LPGs fabricated by different techniques. For LPGs by the arc-discharge method, directional bending sensitivity was observed and resonant peaks were found to exhibit red and blue shifts, depending on the orientation of bends [12]. However, the LPG fabricated by $\mathrm{CO}_{2}$ laser irradiation exhibited resonant mode splitting and orientational dependence due to asymmetrical nature of inscribed gratings [28], which was not observed in SMF. In either case, the bending sensitivity of LPG in PCF was higher than its SMF counterparts, giving a maximal value of $-12.4 \mathrm{~nm}$ and $27.9 \mathrm{~nm}$ for the arc-discharge and $\mathrm{CO}_{2}$ laser irradiation methods, respectively.

LPG is also fabricated in hollow-core PBG PCFs. However, introducing the refractive index modulation into the hollow-core PBG PCF is not easy as most of the mode power propagates in air rather in silica. Wang et al. reported the first LPG in the hollow-core PCFs by using $\mathrm{CO}_{2}$ laser [29]. The resulting LPG is very sensitive to the axial strain $(-830 \mathrm{pm} / \mu \varepsilon)$, which is two orders of magnitude higher than LPG in index-guiding PCF. In the meanwhile, it is insensitive to the temperature $\left(2.9 \mathrm{pm} /{ }^{\circ} \mathrm{C}\right)$, curvature, and surrounding refractive index.

\section{LPG-based devices in PCF}

PCF LPGs can also be used for a variety of in-fiber devices. The resonant wavelength can be tuned thermally, mechanically, or electrically by using the methods that have been applied to SMF-based LPGs, which allows tunable band rejection filter to be implemented as discussed in Section 2. In this section, we discuss devices that may not be easily obtained from SMF-based LPGs.
PDLs are commonly observed in SMF LPGs. However, a much higher PDL over a broad wavelength range has been obtained in PCF LPG. Wang et al. demonstrated an in-fiber polarizer with a polarization extinction ratio of more than $20 \mathrm{~dB}$ over a wavelength range of about $11 \mathrm{~nm}$ near $1550 \mathrm{~nm}$ [30].

The air-holes in PCF facilitate the infiltration of liquid crystal (LC) or refractive index fluid (RIF) $[19,31-33,37]$ into the fiber structure. The introduction of the LC or RIF provides additional degree of freedom for designing novel in-fiber devices. For example, the polarization properties can be thermally tuned through the filled LC, resulting in high polarization dependent loss [32]. The LPG in a fluid-filled PCF has a high thermal tuning coefficient of up to $-1.58 \mathrm{~nm} /{ }^{\circ} \mathrm{C}$, which is due to the large thermo-optic coefficients of fluid [33].

LPG-based in-fiber Mach-Zehnder interferometers (MZI) were also demonstrated in PCF [34-36]. Temperature sensitivity and strain sensitivity of these interferometers have been measured and found to be respectively about 29 times smaller and about 6 times larger than that of an MZI fabricated on a SMF using the same technique [36]. The device can be potentially used as stable multi-channel filter with low temperature dependence.

\section{Conclusions}

In summary, we have reviewed various fabrication techniques for fabricating LPGs in PCFs. The advantages and disadvantages of different techniques have been contrasted. The responses of the LPGs to various parameters are discussed and compared with their SMF counterparts. The PCF-based LPGs have relatively higher sensitivity to strain but lower sensitivity to temperature, which make them an ideal candidate for temperature insensitive strain measurement. The sensor also shows high sensitivity to surrounding refractive index and curvature. We also reviewed in-fiber 
devices based on the LPGs in PCFs. With the unique characteristics of the LPGs in various types of PCFs, it is expected that they would play an important role in the field of fiber optic sensors and devices.

\section{Acknowledgement}

This work was supported by the Research Grants Council of the Hong Kong Special Administrative Region of China through grants PolyU5182/07E and PolyU5176/05E, and the NSFC of China through a grant 60629401.

Open Access This article is distributed under the terms of the Creative Commons Attribution License which permits any use, distribution, and reproduction in any medium, provided the original author(s) and source are credited.

\section{References}

[1] A. M. Vengsarkar, P. J. Lemaire, J. B. Judkins, et al., "Long-period fiber gratings as band-rejection filters," J. Lightwave Technol., vol. 14, no. 1, pp. 58-64, 1996.

[2] T. A. Birks, J. C. Knight, and P. St. J. Russell, "Endlessly single-mode photonic crystal fiber," Opt. Lett., vol. 22, no. 13, pp. 961-963, 1997.

[3] R. F. Cregan, B. J. Mangan, J. C. Knight, et al., "Single-mode photonic band gap guidance of light in air," Science, vol. 285, no. 5433, pp. 1537-1359, 1999.

[4] G. Kakarantzas, T. A. Birks, and P. S. Russell, "Structural long-period gratings in photonic crystal fibers," Opt. Lett., vol. 27, no. 12, pp. 1013-1015, 2002.

[5] Y. N. Zhu, P. Shum, J. H. Chong, et al., "Deep-notch, ultracompact long-period grating in a large-mode-area photonic crystal fiber," Opt. Lett., vol. 28, no. 24, pp. 2467-2469, 2003.

[6] Y. N. Zhu, P. Shum, H. W. Bay, et al., "Strain-insensitive and high-temperature long-period gratings inscribed in photonic crystal fiber," Opt. Lett., vol. 30, no. 4, pp. 367-369, 2005.

[7] L. Rindorf, J. B. Jensen, M. Dufva, et al., "Photonic crystal fiber long-period gratings for biochemical sensing," Opt. Express, vol. 14, no. 18, pp. 8224-8231, 2006.

[8] Y. P. Wang, L. Xiao, D. N. Wang, and W. Jin, "Highly sensitive long-period fiber-grating strain sensors with low temperature sensitivity," Opt. Lett., vol. 31, no. 23, pp. 3414-3416, 2006.

[9] Y. P. Wang, W. Jin, J. Ju, et al., "Long period gratings in air-core photonic bandgap fibers," Opt. Express, vol. 16, no. 4, pp. 2784-2790, 2008.

[10] H. W. Lee, Y. Liu, and K. S. Chiang, "Writing of long-period gratings in conventional and photonic-crystal polarization-maintaining fibers by $\mathrm{CO}_{2}$-laser pulses," IEEE Photon. Technol. Lett., vol. 20, no. 2, pp. 132-134, 2008.

[11] K. Morishita and Y. Miyake, "Fabrication and resonance wavelengths of long-period gratings written in a pure-silica photonic crystal fiber by the glass structure change," J. of Lightwave Technol., vol. 22, no. 2, pp. 625-630, 2004.

[12] H. Dobb, K. Kalli, and D. J. Webb, "Measured sensitivity of arc-induced long-period grating sensors in photonic crystal fiber," Opt. Commun., vol. 260, no. 1, pp. 184-191, 2006.

[13] J. S. Petrovic, H. Dobb, V. K. Mezentsev, et al., "Sensitivity of LPGs in PCFs fabricated by an electric arc to temperature, strain, and external refractive index," J. of Lightwave Technol., vol. 25, no. 5, pp. 1306-1312, 2007.

[14] J. H. Lim, K. S. Lee, J. C. Kim, and B. H. Lee, "Tunable fiber gratings fabricated in photonic crystal fiber by use of mechanical pressure," Opt. Lett., vol. 29, no. 4, pp. 331-333, 2004.

[15] D. Lee, Y. Jung, Y. S. Jeong, et al., "Highly polarization-dependent periodic coupling in mechanically induced long period grating over air-silica fibers," Opt. Lett., vol. 31, no. 3, pp. 296-298, 2006.

[16] K. N. Parka, T. Erdoganb, and K. S. Lee, "Cladding mode coupling in long-period gratings formed in photonic crystal fibers," Opt. Commun., vol. 266, no. 2, pp. 541-545, 2006.

[17] A. Diez, T. A. Birks, W. H. Reeves, B. J. Mangan, and P. S. J. Russell, "Excitation of cladding modes in photonic crystal fibers by flexural acoustic waves," Opt. Lett., vol. 25, no. 20, pp. 1499-1501, 2000.

[18] M. W. Haakestad and H. E. Engan, “Acoustooptic properties of a weakly multimode solid core photonic crystal fiber," J. Lightwave Technol., vol. 24, no. 2, pp. 838-845, 2006.

[19] D. Yeom, P. Steinvurzel, B. J. Eggleton, et al., "Tunable acoustic gratings in solid-core photonic bandgap fiber," Opt. Express, vol. 15, no. 6, pp. 3513-3518, 2007.

[20] K. S. Hong, H. C. Park, B. Y. Kim, et al., “1000 nm tunable acousto-optic filter based on photonic crystal fiber," Appl. Phy. Lett., vol. 92, no. 3, pp. 031110-1-031110-3, 2008.

[21] A. A. fotiadi, G. Brambilla, T. Ernst, S. A. Slattery, and D. N. Nikogosyan, "TPA-induced long-period gratings in a photonic crystal fiber: inscription and temperature sensing properties," J. Opt. Soc. Am. B, 
vol. 24, no. 7, pp. 1475-1481, 2007.

[22] D. D. Davis, T. K. Gaylord, E. N. Glytsis, et al., "Long-period fiber grating fabrication with focused $\mathrm{CO}_{2}$ laser pulses," Electron. Lett., vol. 34, no. 3, pp. 302-303, 1998.

[23] W. Jin, Z. Wang, and J. Ju, "Two-mode photonic crystal fibers," Opt. Express, vol. 13, no. 6, pp. 2082-2088, 2005.

[24] C. L. Zhao, L. Xiao, J. Ju, et al., "Strain and temperature characteristics of a long-period grating written in a photonic crystal fiber and its application as a temperature insensitive strain sensor," J. Lightwave Technol., vol. 26, no. 2, pp. 220-227, 2008.

[25] V. Bhatia and A. M. Vengsarkar, "Optical fiber long-period grating sensors," Opt. Lett., vol. 21, no. 9, pp. 692-694, 1996.

[26] Y. Zhu, Z. He, and H. Du, "Detection of external refractive index change with high sensitivity using long-period gratings in photonic crystal fiber," Sensors and Actuators B, vol. 131, no. 1, pp. 265-269, 2008.

[27] L. Rindorf and O. Bang, "Highly sensitive refractometer with a photonic-crystal-fiber long-period grating," Opt. Lett., vol. 33, no. 6, pp. 563-565, 2008.

[28] Z. He, Y. Zhu, and H. Du, "Effect of macro-bending on resonant wavelength and intensity of long-period gratings in photonic crystal fiber," Opt. Express, vol. 15, no. 4, pp. 1804-1810, 2007.

[29] Y. Wang, W. Jin, J. Ju, et al., "Long period gratings in air-core photonic bandgap fibers," Opt. Express, vol. 16, no. 4, pp. 2784-2790, 2008.

[30] Y. Wang, L. Xiao, D. N. Wang, and W. Jin, "In-fiber polarizer based on a long-period fiber grating written on photonic crystal fiber," Opt. Lett., vol. 32, no. 9, pp. 1035-1037, 2007.

[31] P. Steinvurzel, E. D. Moore, E. C. Mägi,
B. T. Kuhlmey, and B. J. Eggleton, "Long period grating resonances in photonic bandgap fiber," Opt. Express, vol. 14, no. 7, pp. 3007-3014, 2006.

[32]D. Noordegraaf, L. Scolari, J. Lægsgaard, L. Rindorf, and T. T. Alkeskjold, "Electrically and mechanically induced long period gratings in liquid crystal photonic bandgap fibers," Opt. Express, vol. 15, no. 13, pp. 7901-7912, 2007.

[33] P. Steinvurzel, E. D. Moore, E. C. Mägi, et al., "Tuning properties of long period gratings in photonic bandgap fibers," Opt. Lett., vol. 31, no. 14, pp. 2103-2105, 2006.

[34] J. H. Lim, H. S. Jang, K. S. Lee, J. C. Kim, and B. H. Lee, "Mach-Zehnder interferometer formed in a photonic crystal fiber based on a pair of long-period fiber gratings," Opt. Lett., vol. 29, no. 4, pp. 346-348, 2004.

[35] H. Y. Choi, K. W. Park, and B. H. Lee, "Photonic crystal fiber interferometer composed of a long period grating and one point collapsing of air holes," Opt. Lett., vol. 33, no. 8, pp. 812-814, 2008.

[36] J. Ju, W. Jin, and H. L Ho, "Compact in-fiber interferometer formed by long-period gratings in photonic crystal fiber," IEEE Photon. Technol. Lett., vol. 20, no. 23, pp. 1899-1901, 2008.

[37] Y. Wang, "Review of long period fiber gratings written by $\mathrm{CO}_{2}$ laser," J. Appl. Phys., vol. 108, no. 8, pp. 081101-1-081101-18, 2010.

[38] Y. Rao, Y. Wang, Z. Ran, and T. Zhu, "Novel fiber-Optic sensors based on long-period fiber gratings written by high-frequency $\mathrm{CO}_{2}$ laser pulses," J. Lightwave Technol., vol. 21, no. 5, pp. 1320-1327, 2003.

[39] G. Rego, R. Falate, O. Ivanov, and J. L. Santos, "Simultaneous temperature and strain measurements performed by a step-changed arc-induced long-period fiber grating," Appl. Opt., vol. 46, no. 9, pp. 1392-1396, 2007. 